\title{
UNESCO Biosphäre Entlebuch: Modell für eine nachhaltige Regionalentwicklung? Konzept Zielerreichungskontrolle
}

\author{
Annette Schmid, Engelbert Ruoss, Schüpfheim, Hans \\ Elsasser, Zürich

\section{UNESCO Biosphäre Entlebuch: Modell für eine nachhaltige Regionalentwicklung! - Problem- stellung}

Das Entlebuch ist im September 2001 von der UNESCO als erstes Biosphärenreservat der Schweiz gemäss Sevilla-Strategie von 1995 anerkannt worden. Biosphärenreservate sind grossflächige, repräsentative Ausschnitte von Natur- und Kulturlandschaften. Die UNESCO strebt dabei ein weltumspannendes Netz an, welches die verschiedenen Ökosysteme und Naturräume der Erde umfasst. Zur Zeit existieren 440 Biosphärenreservate in 97 Ländern (www.unesco.org/ mab/brlist.htm 3.1.2004). Gemäss Sevilla-Strategie sollen Biosphärenreservate der Umsetzung der Agenda 21 verpflichtet und somit Modellregionen für eine nachhaltige Entwicklung sein (UNESCO 1996).

\section{UNESCO Biosphäre Entlebuch: Modell für eine nachhaltige Regionalentwicklung? - Zielsetzung und Fragestellungen}

Um zu überprüfen, ob die UNESCO Biosphäre Entlebuch (UBE) dem Anspruch einer Modellregion tatsächlich gerecht wird, bedarf es regelmässiger Evaluationen. In einer Forschungsarbeit werden Schwerpunkte, Ziele und Indikatoren einer nachhaltigen Regionalentwicklung hergeleitet, welche als Basis für eine künftige Zielerreichungskontrolle dienen werden. Die Zielerreichungskontrolle untersucht, ob die gesetzten Ziele - unabhängig von den getroffenen Massnahmen - erreicht worden sind. Sie basiert auf einem Vergleich zwischen dem ex ante geplanten Sollwert mit dem ex post tatsächlich gemessenen Istwert des Zielindikators (SPEHL 1981: 20).

Um Schwerpunkte, Ziele und Indikatoren einer nachhaltigen Regionalentwicklung herzuleiten - und damit das Konzept zur Zielerreichungskontrolle in inhaltlicher Hinsicht zu erstellen - müssen Fragen auf verschiedenen Ebenen beantwortet werden:

- institutionelle Ebene: Welche spezifische Bedingungen eines Biosphärenreservats müssen beachten werden?

- regionale Ebene: Welche regionale Voraussetzungen gilt es im Entlebuch zu berücksichtigen?
- konzeptionelle Ebene: Wie soll die Herleitung der Indikatoren strukturiert sein?

- inhaltliche Ebene: Was wird unter einer nachhaltigen Regionalentwicklung im Allgemeinen verstanden, was unter einer nachhaltigen Regionalentwicklung mit Tourismus im Speziellen?

- akteurorientierte Ebene: Wer wird am Aufbau der Zielerreichungskontrolle beteiligt?

- methodische Ebene: Welche Methoden sollen zur Erstellung des Konzepts zur Zielerreichungskontrolle verwendet werden?

Mit der Beantwortung dieser Fragen in Kapitel 3 werden die Anforderungen an den Aufbau des Konzepts zur Zielerreichungskontrolle konkretisiert. In Kapitel 4 wird dann das Konzept am Beispiel des Tourismus vorgestellt, bevor in Kapitel 5 ein Fazit gezogen wird.

\section{Anforderungen an den Aufbau des Konzepts zur Zielerreichungskontrolle}

\subsection{Institutionelle Anforderungen: UNESCO Biosphärenreservate}

Damit eine Region in der Schweiz von der UNESCO als Biosphärenreservat anerkannt werden kann, muss sie die Kriterien für die Anerkennung und Überprüfung von Biosphärenreservaten der UNESCO in der Schweiz (KüTTEl \& RoBIN 2001) erfüllen.

Die UBE repräsentiert eine voralpine Moor- und Karstlandschaft und bedeckt mit $394 \mathrm{~km}^{2}$ knapp 1\% der Schweizer Landesfläche (Regionalmanagement Biosphärenreservat Entlebuch (BRE) 2002b: 3). Biosphärenreservate dienen der Erhaltung der Biodiversität (Schutzfunktion), fördern eine nachhaltige Regionalentwicklung (Entwicklungsfunktion) und unterstützen Demonstrationsprojekte der Umweltbildung, Forschung und Umweltbeobachtung (logistische Funktion) (UNESCO 1996:20). Damit diese Funktionen umgesetzt werden können, wird jedes Biosphärenreservat - abgestuft nach dem Einfluss der menschlichen Tätigkeit - in eine Kern-, eine Pflege- sowie eine Entwicklungszone gegliedert (KüTTEL \& RoBin 2001: 26ff) (Abb. 1):

- Die Kernzone dient der freien Dynamik und Entwicklung der natürlichen und naturnahen Ökosysteme. Die für Grundeigentümer verbindlich geschützten Moorbiotope, das Naturschutzgebiet Schrattenfluh und das Jagdbanngebiet Tannhorn gehören in der UBE zu dieser Zone. Auenlandschaften von nationaler Bedeutung sowie Waldreservate 

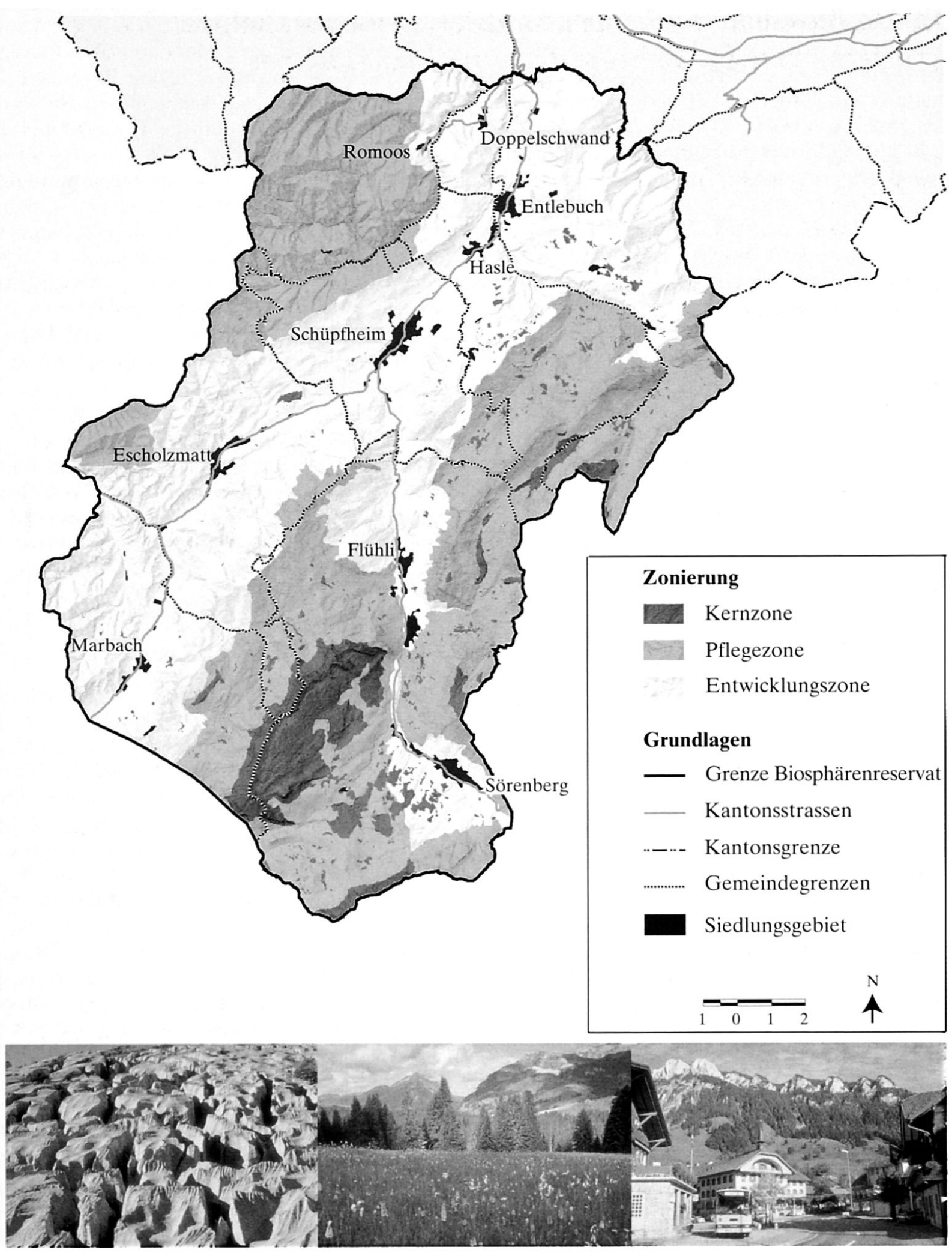

Abb. 1: Zonierung der UNESCO Biosphäre Entlebuch (UBE) (Foto 1: Kernzone: Naturschutzgebiet Schrattenfluh; Foto 2: Pflegezone: Moorlandschaft Habkern/Sörenberg; Foto 3: Entwicklungszone: Dorfzentrum Flühli) Zonation of UNESCO Biosphere Reserve Entlebuch (UBE) (Photo 1: Core Area: nature conservation area of Schrattenfluh; Photo 2: Buffer Zone: moorlands of Habkern/Sörenberg; Photo 3: Transition Area: centre of Flühli)

Zonage de "UNESCO Biosphère Entlebuch» (UBE) (Photo 1: Aire centrale: réserve naturelle protégée Schrattenfluh; Photo 2: Zone tampon: Paysage marécageux Habkern/Sörenberg; Photo 3: Aire de transition: centre villageois de Flïhli) Quelle: GIS-Koordinationsstelle Luzern; Foto 1: T. Schnider, Foto 2: A. Huber, Foto 3: T. SchNider 
werden diese Zone später ergänzen. Die Kernzone umfasst in der UBE $8 \%$ der Fläche.

- Die Pflegezone umgibt die Kernzone und dient der Erhaltung und Förderung der traditionellen Kulturlandschaft. Sie bedeckt $42 \%$ der Fläche der UBE und beinhaltet Moorlandschaften sowie Landschaften und Naturdenkmäler von nationaler Bedeutung.

- Die Entwicklungszone ist Lebens-, Wirtschafts- und Erholungsraum der Menschen und ist mit 50\% der Gesamtfläche die grösste Zone.

Kern- und Pflegezonen umfassen zusammen $50 \%$ der Fläche der UBE. Damit kommt dem Schutzaspekt ein grosses Gewicht zu, müssen doch gemäss Kriterien nur $20 \%$ der Gesamtfläche in der Kern- und Pflegezone liegen. Jedes Biosphärenreservat muss über ein Biosphärenmanagement verfügen. Dieses ist zuständig für Information, Koordination, Animation und Erfolgskontrolle. Gemeinsam mit der im Gebiet lebenden Bevölkerung sollen beispielhafte Konzepte zu Schutz, Pflege und nachhaltiger Entwicklung in partizipativen Prozessen erarbeitet und umgesetzt werden. Die Organisationsstruktur der UBE spiegelt deutlich wider, dass die Partizipation der Bevölkerung ein wichtiges Anliegen ist. So liegt die strategische und operative Leitung der UBE in den Händen der Ortsansässigen. Zudem konnte die Bevölkerung im Jahr 2000 zum ersten Mal in der Geschichte der Biosphärenreservate an Gemeindeversammlungen abstimmen, ob sie dem weltweiten Netz der Biosphärenreservate beitreten will. Mit einem unerwartet hohen Ja-Stimmenanteil von $94 \%$ steht die Bevölkerung klar hinter dem Projekt (Regionalmanagement BiosphärenReservat ENTLEBUCH (BRE) 2002a: 8).

\subsection{Regionale Anforderungen: Entlebuch als ländliche Region}

In den acht Luzerner Gemeinden Doppleschwand, Entlebuch, Escholzmatt, Flühli, Hasle, Marbach, Romoos und Schüpfheim leben Ende 2001 16'682 Einwohner. Von den gut 8 '000 Beschäftigten sind $36.5 \%$ im ersten, $23.6 \%$ im zweiten und $39.9 \%$ im dritten Sektor tätig, wobei der Tourismus ein wichtiger Arbeitgeber ist (Амт Für STATISTIK (AfS) 2003: 563). Im Vergleich zur Schweiz (1. Sektor: $5.8 \%$, 2. Sektor 26.6\%, 3. Sektor 67.6\%; Amt Für Statistik (AfS) 2003: 105) ist die Sektoralstruktur des Entlebuchs damit noch sehr stark durch den ersten Sektor geprägt. Wie für den ländlichen Raum typisch, ist das Entlebuch äusserst reich an geschützten Natur- und Kulturlandschaften, welche nun die Kern- und Pflegezone des Biosphärenreservats bilden. Obwohl die Ausscheidung dieser Gebiete nach natur- und landschaftsschutzpolitischen Kriterien vorgenommen worden ist, konnte gezeigt werden, dass diese aus der Sicht der Bevölkerung auch die schönsten Landschaften der Region sind (WALTHER 2000). Dies ist für den Tourismus von grösster Bedeutung.
Im Sommer wird die Region denn auch als Wander- und Bikegebiet präsentiert. Markierte Wanderwege und Bikerouten stehen zur Verfügung. Besondere Bedeutung kommt schon seit längerem den Naturerlebnissen zu, welche das natürliche Potenzial der Region auf sanfte Art und Weise in Wert setzen. So wurden bereits ein provisorisches Naturinfozentrum aufgebaut, 7 Lehrpfade ausgeschildert und diverse Exkursionen angeboten, bevor die Region als Biosphärenreservat anerkannt worden ist. Im Winter hat sich die Region dem Schneesport verschrieben. Die touristische Infrastruktur umfasst 4 Bergbahnen, 25 Skilifte sowie 3 Kinderskilifte. 19 Anlagen befinden sich im Wintersportort Sörenberg, 5 in Marbach (www.biosphaere.ch/pages/ a_region /a2_1.html 4.1.2002).

Auf der Nachfrageseite verzeichnet die Region im Jahr 2000 knapp 450'000 Logiernächte. Davon fallen 7\% auf die Hotellerie, $4 \%$ auf die Parahotellerie und 52\% auf den Eigenheimtourismus. Die räumliche Verteilung des Tourismus ist zur Zeit einseitig, entfallen doch von den ausgewiesenen Logiernächten $97 \%$ auf die Gemeinde Flühli Sörenberg (Regionalmanagement Biosphärenreservat Entlebuch (BRE) 2001: 32). Der Winter gilt als stärkste Saison.

\subsection{Konzeptionelle Anforderungen: Leitbildorien- tierte Entwicklung von Indikatoren}

Die Zielerreichungskontrolle basiert auf Indikatoren, welche in Anlehnung an die «leitbildorientierte Entwicklung von Indikatoren" (LOEI) des SACHVERSTÄNDIGENRATS FÜR UMWELTFRAGEN (SRU) in Deutschland (1994: 101) hergeleitet werden: Ausgangspunkt der LOEI ist ein Leitbild, welches definiert, was unter einer nachhaltigen Regionalentwicklung mit Tourismus verstanden wird. Darauf aufbauend wird ein Analyserahmen erstellt, welcher alle relevanten Themen im Zusammenhang mit einer nachhaltigen Regionalentwicklung mit Tourismus im Allgemeinen nennt und strukturiert (BosshaRd 1997: 6). Innerhalb dieses Analyserahmens müssen Schwerpunkte für die Region bestimmt, Ziele gesetzt und Indikatoren zu deren Überprüfung gewählt werden. Der Prozess der Indikatorenentwicklung enthält mehrere Rückkopplungsschleifen und ist zirkulär angelegt. Dies kommt der Interpretation einer nachhaltigen Entwicklung als einem langfristigen Lernprozess entgegen.

\subsection{Inhaltliche Anforderungen: Nachhaltige Regionalentwicklung mit Tourismus}

Biosphärenreservate sollen Modelle für eine nachhaltige Regionalentwicklung sein. In Anlehnung an Peters et al. (1996) werden in der UBE ins Zentrum einer nachhaltigen Regionalentwicklung sogenannte Produktlinien gestellt: Sie ist definiert als Lebensweg eines Produkts entlang den Stationen Rohstoffgewinnung, Transport, Be- und Verarbeitung, Handel, Nut- 
zung und Entsorgung/Wiederverwertung. Im Zusammenhang mit einer nachhaltigen Entwicklung werden diese Produktlinien unter den drei Gesichtspunkten Umwelt, Wirtschaft und Gesellschaft analysiert: Dabei werden die Produktlinien

- in ökologischer Hinsicht als Stoffströme,

- in ökonomischer Hinsicht als Wertschöpfungsketten und

- in sozialer Hinsicht als Handlungsketten der an der Produkterstellung Beteiligten und von den Auswirkungen der Produkterstellung Betroffenen verstanden.

In einer nachhaltigen Regionalentwicklung gilt es:

- Stoffströme kleinräumig zu führen und Kreisläufe weitgehend zu schliessen;

- Wertschöpfungsketten regional zu schliessen und einen Beitrag an die Grundbedarfsversorgung zu leisten und

- Handlungsketten durch Kooperationen aufzubauen. In einer nachhaltigen Regionalentwicklung mit Tourismus wird die Produktlinie bewusst aus der Sicht der Gäste formuliert. Sie interessieren sich nicht für Einzelprodukte sondern für ein ganzes Leistungsbündel. Die Produktlinie im Tourismus umfasst im Wesentlichen die Bausteine Information, Anreise, Unterkunft, Verpflegung, Aktivitäten, Mobilität in der Region sowie Abreise (Bieger \& Laesser 1998: 27). Diese Bausteine werden von unabhängigen Anbietern erstellt und müssen deshalb in der Region koordiniert werden. Diese Aufgabe übernimmt die Destination. Das touristische Leistungsbündel hat wiederum Auswirkungen auf die Dimensionen einer nachhaltigen Entwicklung, welche beachtet werden müssen.

Dieses nachhaltige touristische Leistungsbündel übernimmt in der LOEI die Funktion des Analyserahmens. Innerhalb des Bündels gilt es nun, regionsspezifische Schwerpunkte, Ziele und Indikatoren zu bestimmen.

\subsection{Akteurorientierte Anforderungen: Entlebuch Tourismus als Kooperationspartner}

Nachhaltige Entwicklung ist ein normativer Ansatz, welcher von Werten und Normen der Beteiligten abhängt (THIERSTEIN \& WALSER 2000: 52ff.). So verlangt auch die Sevilla-Strategie, dass die lokale Bevölkerung an der Ausgestaltung des Biosphärenreservats partizipieren kann (UNESCO 1996: 10f.). Gemäss KrÜGER \& LOHNERT (1996: 45) wird Partizipation im Zusammenhang mit Entwicklungsfragen auf regionaler und lokaler Ebene als «Teilnahme und Teilhabe der betroffenen Bevölkerung an räumlich begrenzten Entwicklungsprozessen» verstanden. In der Literatur werden verschiedene Partizipationsformen unterschieden. Gemeinsam ist diesen Ansätzen, dass Partizipation als Kontinuum verstanden (United Nations Developement Programme (UNDP), Civil Society OrganiZations and Participation Programme (CSOPP) 1998:
Kap. 2) und anhand verschiedener Stufen fassbar gemacht wird. So unterscheiden Bischoff, Selle \& SINNING (1995: 18f.) mit dem Erkunden von Interessen, Information, Beteiligung und Kooperation beispielsweise vier Stufen. Grundsätzlich gilt, dass keine Stufe als die beste bezeichnet werden kann, sondern der Partizipationsgrad den jeweiligen Verhältnissen angepasst werden muss (RAMSAUER 2000: 20).

In der UBE wird zur Erstellung des Konzepts zur Zielerreichungskontrolle die Stufe der Kooperation, verstanden als «Zusammenarbeit selbstständiger Akteure» (Selle 1997: 11), angestrebt. Die Akteure werden somit bereits in die Planung einbezogen, denn hier wird entschieden, welche Parameter zukünftig evaluiert werden sollen. Auch die spätere Durchführung der Evaluation sowie die Nutzung der Resultate soll gemeinsam mit den Akteuren geschehen. Der Einbezug von bereits bestehenden Institutionen ist wichtig, denn der Aufbau von parallelen Partizipationsstrukturen ist nicht wünschenswert und resultiert in ineffektiven und nicht repräsentativen Körperschaften (UNDP, CSOPP 1998: Kap. 1.3). In der UBE wurde zur Erstellung des Konzepts zur Zielerreichungskontrolle mit der regionalen Tourismusorganisation Entlebuch Tourismus (ET), in der alle Gemeinden der Region vertreten sind, kooperiert. Damit die gesamte touristische Anbieterseite einbezogen werden konnte, wurden neben dem Vorstand von ET und dem Biosphäremanagement weitere Personen gezielt eingeladen, so dass 27 Personen am Aufbau des Konzepts beteiligt waren.

\subsection{Methodische Anforderungen: Moderierte Workshops}

Um die regionalen Akteure einzubeziehen, wurde ein moderierter Workshop durchgeführt, da er sich für die Arbeit in und mit Gruppen gut eignet (BorTz \& Döring 2002: 320; Neuland 1999: 3). Moderierte Workshops erlauben es, implizites Wissen, welches an Personen und ihren sozialen Kontext gebunden ist, in interaktiven und kooperativen Lernprozessen zugänglich zu machen (MORGaN 1997: 493). Dieses «Learning by interacting» oder «Learning by networking” (BUTZIN 2000: 155) kommt der Interpretation einer nachhaltigen Entwicklung als einem langfristigen Lernprozess, welcher auf Partizipation und Netzwerken beruht (THIERSTEIN \& WALSER 2000:55ff.), entgegen.

Ziel des Workshops war die Herleitung von Schwerpunkten, Zielen und Indikatoren der zukünftigen Zielerreichungskontrolle. Der Workshop umfasste zwei Hauptteile:

- Im ersten Teil wurde die aktuelle und zukünftige Lage im Tourismus in der UBE präsentiert und diskutiert. Dazu war bei den Teilnehmenden des Workshops im Voraus eine schriftliche Befragung 
zu den Stärken, Schwächen, Chancen und Gefahren im Tourismus durchgeführt worden (EUROPEAN Commission 1999: 41ff.). Mit dieser SWOT-Analyse (strengths, weaknesses, opportunities, threats) konnte ein gemeinsames Problemverständnis erarbeitet werden.

- Der zweite Teil war der Lancierung des Konzepts zur Zielerreichungskontrolle gewidmet. So galt es in einem ersten Schritt innerhalb des Analyserahmens Schwerpunkte zu bestimmen. Anschliessend wurden zu den gewählten Schwerpunkten Ziele definiert und Indikatoren zu deren Überprüfung festgelegt. Als Arbeitsformen in diesem Workshopteil dominierten Gruppenarbeiten und Plenumsdiskussionen. Die wichtigste Moderationstechnik war die Kartenabfrage (NEuland 1999).

\section{Konzept Zielerreichungskontrolle}

Nachdem in Kapitel 3 die Anforderungen an den Aufbau des Konzepts zur Zielerreichungskontrolle konkretisiert wurden, erfolgt nun in Kapitel 4 die Präsentation dieses Konzepts. Aus der leitbildorientierten Entwicklung von Indikatoren werden die gewählten regionsspezifischen Schwerpunkte des Analyserahmens vorgestellt (Abb. 2). Dabei werden zuerst einige Ausführungen zur Destination UBE gemacht, da sie für die Koordination des gesamten touristischen Leistungsbündels verantwortlich ist. Anschliessend werden die einzelnen Bausteine des Bündels vorgestellt sowie die Dimensionen einer nachhaltigen Entwicklung thematisiert. In der Zwischenzeit stehen zu einigen der Schwerpunkte bereits Daten zur Verfügung, welche hier in die Präsentation einfliessen werden. Sie bilden erste Umsetzungen des Konzepts zur Zielerreichungskontrolle in der Praxis.

\subsection{Destination UBE}

Zur Zeit konzentriert sich der Tourismus räumlich hauptsächlich auf die Gemeinde Flühli-Sörenberg (Kap. 3.2). In Zukunft sollen aber alle Gemeinden ins touristische Angebot der UBE einbezogen werden. Deshalb wird die Destination «UNESCO Biosphäre Entlebuch» aufgebaut. Das Label Biosphärenreservat gilt in der Schweiz als klare Differenzierungsstrategie und lässt eine Positionierung der Destination im Markt zu. Auf die Sommersaison 2004 sollen eine regionale Geschäftsstelle eröffnet sowie eine Ombudsstelle eingerichtet werden, welche bei allfällig auftretenden Konflikten zwischen den verschiedenen Tourismuspartnern schlichtend eingreifen kann.

\subsection{Bausteine des touristischen Leistungsbündels}

$\mathrm{Zu}$ den verschiedenen Bausteinen des touristischen Leistungsbündels wurden in den Workshops folgende Schwerpunkte bestimmt:
- Information: Das Destinationsmarketing soll gemäss dem sogenannten «4 P's Model» von McCarthy alle vier Marketinginstrumente - product, price, place, promotion - umfassen (THOMmen 1992: 227): So sollen touristische Angebote regional koordiniert (z.B. regionales Wanderbuch), eine zeitliche und zielgruppenspezifische Preisdifferenzierung vorgenommen, eine gemeinsame Vertriebspolitik angestrebt (z.B. Aufbau von regionalen und lokalen Tourismusbüros) sowie eine gemeinsame Kommunikationspolitik betrieben werden. Bei letzterer spielt die Broschüre «Erlebnis UBE» eine wichtige Rolle, gibt sie doch einen Hinweis auf die zunehmende Bedeutung der Destination UBE: 1997 lief die Broschüre unter dem Namen "Sörenberg Flühli», 1998 und 1999 unter «Sörenberg Entlebuch», 2000 unter «Entlebuch Sörenberg» und ab 2001 unter «Biosphärenreservat Entlebuch». Als Basis des Marketings soll eine gemeinsame Marktforschung betrieben werden.

- An- und Abreise: Eine nachhaltige Verkehrspolitik benutzt die "Triple S-Strategie», in der in erster Priorität Verkehr vermieden (s saving), in zweiter Priorität der motorisierte Individualverkehr auf den öffentlichen Verkehr verlagert (s hifting) und erst in letzter Priorität eine verträgliche Gestaltung des verbleibenden Verkehrs angestrebt wird ( $\underline{s}$ moothing) (Walter \& Spillmann 1999: 98). Bei der Vermeidungsstrategie steht in der UBE primär die Erhöhung der Aufenthaltsdauer der Gäste im Zentrum, da der Tourismus per Definition auf Verkehr angewiesen ist. Bei der Verlagerungsstrategie gilt es, den Modal-Split zugunsten des Öffentlichen Verkehrs zu erhöhen. Eine erste Erhebung zeigt, dass sich der heutige Modal-Split der Gäste der UBE auf $71 \%$ Personenwagen, $22 \%$ öffentliche Verkehrsmittel, 4\% Motorrad, 2\% Fahrrad und 1\% Car aufteilt (Bollhalder 2000: 133). Zur Verlagerung ist beim Aufenthaltstourismus ein Pilotprojekt zum Tür-zu-Tür-Gepäckservice lanciert, beim Tagestourismus sollen die package-Angebote im snow'n rail (Kombination Skitageskarte und ÖV-An- und Abreise) verstärkt und im rail away (Kombination Erlebnisangebot und ÖV-An- und Abreise) neu lanciert werden. Mobilitätsketten sollen bezüglich Schnelligkeit und Zuverlässigkeit von Bahn- und Busanschlüssen optimiert werden. Gemäss Nationalem Forschungsprogramm «Verkehr und Umwelt» (NFP 41) bringt eine Verkürzung der Reisezeit und eine Verbesserung des Komforts (weniger Umsteigen, Gepäckservice) die Reisenden am ehesten zum Umsteigen (Meier 2000: 59). Zur verträglichen Gestaltung des verbleibenden Verkehrs können Massnahmen im Bereich der Telematik wie das Parkleitsystem in Sörenberg zum Einsatz kommen. Um die genannten Aktivitäten zu koordinieren, wurde im Workshop die Idee einer Mo- 


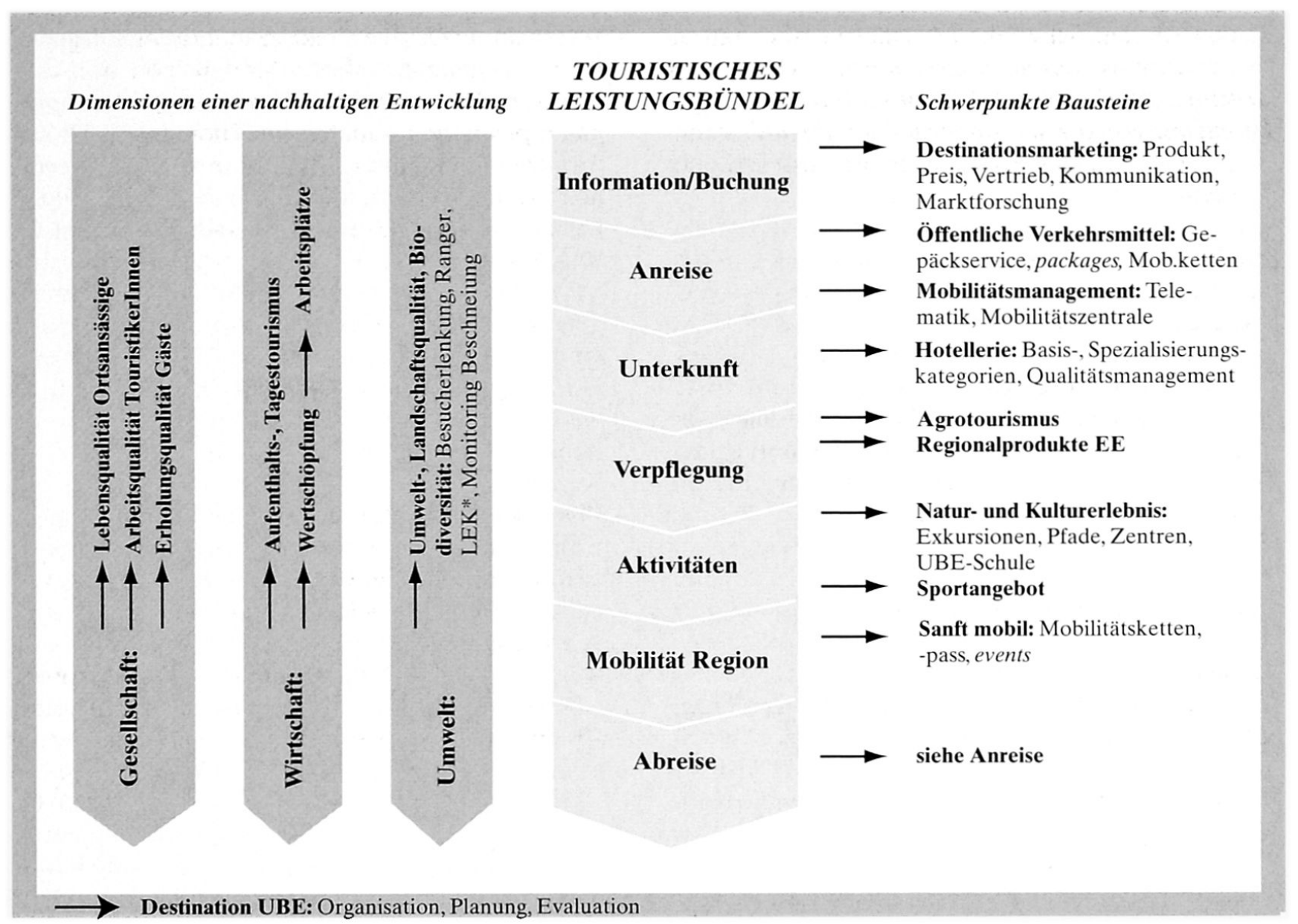

* Landschaftsentwicklungskonzept

Abb. 2: Schwerpunkte Tourismus in der UNESCO Biosphäre Entlebuch (UBE)

Main areas of tourism in UNESCO Biosphere Reserve Entlebuch (UBE)

Centre de gravité "tourisme» dans la réserve de biosphère Entlebuch de l'UNESCO (UBE)

Quelle: eigener Entwurf

bilitätszentrale genannt. Sie kann das Mobilitätsmanagement in der UBE übernehmen.

- Unterkunft:Bei der SWOT-Analyse wurde die Hotellerie als Schwäche bezeichnet, weshalb sie nun gestärkt werden soll. Dabei wird ein qualitatives, ausgewogenes und zielgruppenspezifisches Hotelangebot angestrebt. Um die Qualität, welche neben der nachhaltigen Entwicklung die letzten Jahre in der Tourismusdiskussion prägte (CANOVA 1997: 12), zu erhöhen, soll dem Qualitätsgütesiegel von Schweiz Tourismus zum Durchbruch verholfen werden. Langfristig muss auch eine ausgewogene Struktur der Basiskategorien angestrebt werden - heute gibt es in der UBE kein Vier- oder Fünfstern-Hotel. Spezialisierungen der Hotels, die im Zusammenhang mit der Positionierung der Destination UBE stehen, sind erwünscht (z.B. familienfreundliches Hotel, Öko-, Wanderhotel).
Neben der Hotellerie soll auch die Landwirtschaft vermehrt ins touristische Übernachtungsangebot einbezogen werden. Eine erste Erhebung zeigt, dass von den über 1'000 Landwirtschaftsbetrieben in der Region heute lediglich 34 im Agrotourismus tätig sind, 24 davon im Bereich der Übernachtung (RITTER 2002: Anhang).

- Verpflegung: In der UBE werden Regionalprodukte gefördert und mit der Herkunftsmarke ECHT ENTLEBUCH (EE) ausgezeichnet. Die Marke wurde im Jahr 2002 lanciert und dient der Auszeichnung und unterstützenden Vermarktung von qualitativ guten Produkten, Dienstleistungen und touristischen Angeboten aus dem Entlebuch. Im Zusammenhang mit dem Tourismus sind die Gastro-Partnerbetriebe von Bedeutung. Sie müssen zwei zentrale Voraussetzungen erfüllen: Einerseits müssen sie zertifizierte EE-Produkte oder Produkte aus anderen 
UNESCO Biosphärenreservaten verwenden und deklarieren und anderseits gesamtbetriebliche Voraussetzungen erfüllen. Dazu gehört u.a., dass der Betrieb ein regionstypisches, resp. UBE-spezifisches Angebot führt, dass Mitarbeitende Auskunft über die Region, zu regionalen Veranstaltungen und zur UBE erteilen können und dass Informationsmaterial aufgelegt wird (MARKENKOMMISSION EE 2003). Bis im Februar 2004 konnten 6 Restaurants und Hotels als Partner-Betriebe ausgezeichnet werden.

- Aktivitäten: Im Workshop kam klar zum Ausdruck, dass das Angebot auf den Stärken der Region basieren soll. Entsprechend werden die Natur- und Kulturerlebnisse als Chancen eingestuft. Ein Bestandteil dieser Erlebnisse sind die Exkursionen, welche in Zukunft gestärkt, räumlich auf die gesamte Region und zeitlich auf die Wintersaison ausgedehnt werden sollen. Die Exkursionen werden bereits heute jährlich evaluiert. In der Sommersaison 2002 wurden 61 Exkursionen offiziell ausgeschrieben. Davon konnten 39 mit 474 teilnehmenden Personen durchgeführt werden. Seit 1999 stieg das Exkursionsangebot von 29 auf 61 Exkursionen, die Zahl der Teilnehmenden von 289 auf 474, was einer Zunahme von $64 \%$ entspricht. Den Gesamteindruck der Exkursionen beurteilten 99\% der Teilnehmenden als gut $(28 \%)$ bis sehr gut $(71 \%)$ (Maldı 2003). Für Geschäfts- und Vereinsausflüge sowie Schulen werden immer mehr Sonderexkursionen nach Wunsch ausserhalb des offiziellen Programms angeboten, welche direkt über die UBE organisiert werden. Dieses Segment überstieg mit 66 Exkursionen und 1'475 Teilnehmenden im Jahr 2002 die offiziell ausgeschriebenen Exkursionen erheblich (Auswertungen BIOSPHÄRENMANAGEMENT UBE).

Während auf den Exkursionen die Informationen von Fachpersonen vermittelt werden, können auf den Erlebnispfaden Informationen selber erarbeitet werden. Zur Zeit existieren in der UBE 10 Erlebnispfade und 3 thematische Weitwanderwege wie der Moorlandschaftspfad. Das Bildungsangebot wird durch Erlebniszentren ergänzt. Sie verbessern das begrenzte Indoorangebot in der UBE, welches als Schwäche bezeichnet worden ist. Als neustes Bildungsprojekt gilt die Biosphärenschule. Sie vermittelt Kindern und Jugendlichen die Thematik der nachhaltigen Regionalentwicklung im Feld. Sie wohnen auf Bergbauernhöfen, womit für die Landwirte im Bereich Agrotourismus ein Zusatzeinkommen generiert werden kann. Im Sommer 2003 wurde ein erstes Pilotlager durchgeführt.

Die genannten Natur- und Kulturerlebnisse erlauben es, die gesamte Destination UBE ins touristische Angebot einzubeziehen. Neben diesen Natur- und Kulturerlebnissen soll ein zielgruppenspezifisches Sportangebot angestrebt werden.

- Mobilität Region: In der Region sollen alle wichtigen touristischen Gebiete ohne eigenes Auto bequem erreichbar sein. Dies ist das Ziel des Pilotprojekts «New Mobility: Flühli Sörenberg sanft mobil» (SCHNider 2003). Dazu wird primär auf den ÖV und sekundär auf ÖV-ergänzende Strategien (z.B. car sharing, Vermietung Elektro bikes) zurückgegriffen. Als langfristige Option wurde die Idee eines UBE-Mobilitätspasses genannt. Für grössere events sollen jeweils Mobilitätskonzepte erarbeitet werden, damit kein eigenes Auto zur An- und Abreise nötig ist.

\subsection{Dimensionen einer nachhaltigen Entwicklung}

Im Sinne eines ganzheitlichen Ansatzes werden im Bereich Umwelt die Erhaltung der Umwelt- und Landschaftsqualität und der Biodiversität mittels Besucherlenkung, Rangerwesen, Landschaftsentwicklungskonzept und Monitoring der Beschneiung angestrebt. Im Bereich Wirtschaft soll der Aufenthaltstourismus gefördert und der Tagestourismus konsolidiert werden, um Wertschöpfung und Arbeitsplätze in der Region zu generieren. Im Bereich Gesellschaft stehen bei der Gestaltung der Tourismusentwicklung primär und dauerhaft die Steigerung der Lebensqualität der Ortsansässigen sowie der Arbeitsqualität der Touristiker und sekundär und temporär der Erholungsqualität der Gäste im Zentrum.

\section{UNESCO Biosphäre Entlebuch: Modell für eine nachhaltige Regionalentwicklung!? - Fazit}

Das Konzept der nachhaltigen Entwicklung stellt an den Aufbau der Zielerreichungskontrolle vielfältige Anforderungen, welche sowohl die Prozess- als auch die Inhaltsebene betreffen.

Auf der Prozessebene muss festgehalten werden, dass nachhaltige Entwicklung ein normatives Konzept ist, das von den Werten und Normen der Beteiligten abhängt. Somit galt es, regionale Akteure mittels partizipativer Methoden in die Erarbeitung des Konzepts einzubeziehen. Zudem wird nachhaltige Entwicklung als langfristiger Lernprozess definiert, der auf Partizipation und Netzwerken beruht. Bei partizipativen Evaluationsansätzen steht dabei das Lernen in Netzwerken im Vordergrund, welches in moderierten Workshops zum Tragen kommen kann.

Auf der inhaltlichen Ebene verfolgt das Konzept der nachhaltigen Entwicklung mit der Beachtung von Umwelt, Wirtschaft und Gesellschaft einen ganzheitlichen Ansatz. Damit dieser ganzheitliche Ansatz respektiert werden kann, wird zur Strukturierung einer 
nachhaltigen Regionalentwicklung mit Tourismus das nachhaltige touristische Leistungsbündel als Analyserahmen verwendet. Diesem Rahmen kommt bei der leitbildorientierten Entwicklung von Indikatoren eine zentrale Rolle zu, denn er erlaubt es, eine Verknüpfung der theoretischen Aspekte einer nachhaltigen Regionalentwicklung mit den regionalen Gegebenheiten vorzunehmen. Zudem können neue Ideen implementiert und alte, bereits umgesetzte Massnahmen integriert werden. Der Rahmen gewährt auch für die Zukunft genügend Flexibilität, lassen sich doch Neuerungen problemlos einfügen. Diese Offenheit und Flexibilität kann als Stärke bezeichnet werden. Dies ist im Zusammenhang mit der Definition einer nachhaltigen Entwicklung als langfristigem Lernprozess wichtig. Während aufgrund des Lernprozesses Schwerpunkte, Ziele und Indikatoren überprüft und angepasst werden müssen, bleibt der Rahmen im Wesentlichen unverändert. Er lässt sich somit auf andere Regionen übertragen und ist verallgemeinerbar.

Erste Schritte auf dem Weg zu einer nachhaltigen Regionalentwicklung mit Tourismus können in der UBE ausgewiesen werden. Weitere Anstrengungen sind aber notwendig. Die konsequente Durchführung der Zielerreichungskontrolle wird zeigen, ob die UBE tatsächlich als Modell für eine nachhaltige Regionalentwicklung gelten kann.

\section{Literatur}

Amt Für Statistik (AFS) (2003): Statistisches Jahrbuch des Kantons Luzern 2003. - Luzern.

Bieger, T. \& C. LAesser (Hrsg.) (1998): Neue Strukturen im Tourismus - Der Weg der Schweiz. - Bern: Paul Haupt Verlag.

Bischoff, A., Selle, K. \& H. Sinning (1995): Informieren, Beteiligen, Kooperieren. Kommunikation in Planungsprozessen. Eine Übersicht zu Formen, Verfahren, Methoden und Techniken. - Dortmund: Dortmunder Vertrieb für Bau- und Planungsliteratur.

Bollhalder, E. (2000): Das Potential des nachhaltigen Tourismus im zukünftigen Biosphärenreservat Entlebuch. Eine empirische Untersuchung bei Touristinnen und Touristen in der Region Entlebuch. - Unveröffentlichte Diplomarbeit, Geographisches Institut der Universität Freiburg.

BorTZ, J. \& N. DöRING (2002): Forschungsmethoden und Evaluation für Human- und Sozialwissenschaftler. - Berlin: Springer.

Bosshard, A. (1997): Methodologische und inhaltliche Grundlagen zur Bewertung und Evaluation der Nachhaltigkeit von Landnutzungssystemen (Teil 1). In: Bosshard, A. et al.: Nachhaltige Landnutzung in der Schweiz: Konzeptionelle und inhaltliche Grundlagen für ihre Bewertung, Umsetzung und Evaluation. Bern: 1-13.
Butzin, B. (2000): Netzwerke, Kreative Milieus und Lernende Region. - In: Zeitschrift für Wirtschaftsgeographie 44, 3/4: 149-166.

Canova, L. (1997): Nachhaltigkeit und Tourismusqualität - zwei Seiten einer Medaille. - In: Nachhaltiger Tourismus - Schlüssel zum Erfolg. Tagungsbericht zum ECOTRANS Forum ITB '97. - Berlin: 12-13.

European Commission (1999): Evaluating socio-economic programmes: Principal evaluation techniques and tools. $-=$ MEANS Collection Volume 3, Luxembourg: 1-237.

KrÜGER, F. \& B. LOHNERT (1996): Der Partizipationsbegriff in der geographischen Entwicklungsforschung: Versuch einer Standortbestimmung. - In: Geographische Zeitschrift 84, 1: 43-53.

KütTel, M. \& K. Robin (2001): Kriterien für die Anerkennung und Überprüfung von Biosphärenreservaten der UNESCO in der Schweiz. - = Vollzug Umwelt, Bern: 1-59.

Malli, H. (2003): Auswertung Exkursionsangebot Sommer/Herbst 2002. - Trimmstein: UNESCO Biosphäre Entlebuch (Manuskript).

Markenkommission EE (2003): Reglement Gastro-Partner «echt entlebuch» (EE). - Schüpfheim: UNESCO Biosphäre Entlebuch (Manuskript).

MEIER, R. (2000): Nachhaltiger Freizeitverkehr.-Chur: Rüegger.

MorGan, K. (1997): The Learning Region: Institutions, Innovation and Regional Renewal. - In: Regional Studies 31, 5: 491-503.

Neuland, M. (1999): Neuland-Moderation. - Künzell: Neuland.

Peters, U. et al. (1996): Nachhaltige Regionalentwicklung - ein neues Leitbild für eine veränderte Struktur- und Regionalpolitik. Eine exemplarische Untersuchung an zwei Handlungsfeldern der Region Trier. Trier: Universität Trier [Taurus-Institut].

RamSAUER, M. (2000): Bewertung von Entwicklungsinterventionen. Umsetzung partizipativer Evaluationsmethoden am Beispiel eines Entwicklungsprojektes im Hochland Ecuadors. - Unveröffentlichte Diplomarbeit, Geographisches Institut der Universität Zürich.

REgIONALMANAGEMENT BIOSPHÄRENRESERVAT ENTLEBUCH (BRE) (Hrsg.) (2001): Formulaire de proposition de réserve de biosphère. - Schüpfheim: Biosphärenreservat Entlebuch (Manuskript).

Regionalmanagement BiosphärenRESERVAT ENTLEBUCH (BRE) (Hrsg.) (2002a): Biosphärenreservat Entlebuch Schweiz:erhalten und entwickeln.-Schüpfheim: Regionalmanagement Biosphärenreservat Entlebuch (BRE).

Regionalmanagement BiosphärenReservat EntleBUCH (BRE) (Hrsg.) (2002b): Das Modell Entlebuch. Grobkonzept Biosphärenreservat Entlebuch Januar 2002. - = Berichte aus der Region Entlebuch 2, Schüpfheim: 1-57.

RitTer, Y. (2002): Konzept für den Agrotourismus 
im Biosphärenreservat Entlebuch. - Unveröffentlichte Diplomarbeit, Schweizerische Hochschule für Landwirtschaft, Zollikofen.

SCHNIDER, T. (2003): New Mobility - sanft mobil. Workshop 25. März 2003. - Schüpfheim: UNESCO Biosphäre Entlebuch (Manuskript).

SElle, K. (1997): Planung und Kommunikation. Anmerkungen zur Renaissance eines alten Themas. - In: DISP 129: 40-47.

SPEHL, H. (1981): Regionale Wirtschaftspolitik und regionale Entwicklungsplanung in strukturschwachen Regionen - Erfolgskontrolle und alternative Entwicklungskonzeptionen. - In: AberLe, G. et al. (Hrsg.): Regionalpolitik im Wandel: Beiträge zur Bewertung und zur Koordinierung raumwirksamer Politikbereiche. - = Kleine Schriften der Gesellschaft für Regionale Strukturentwicklung, Bonn: 16-57.

SACHVERSTÄNDIGENRAT FÜR UMWELTFRAGEN (SRU) (1994): Umweltgutachten 1994: Für eine dauerhaft umweltgerechte Entwicklung. - Stuttgart: Metzler-Poeschel.

Thierstein, A. \& M. Walser (2000): Die nachhaltige Region. Ein Handlungsmodell. $-=$ Schriftenreihe des Instituts für Öffentliche Dienstleistungen und Tourismus, Beiträge zur Regionalwirtschaft 1, Bern: 1-264.

Thommen, J.-P. (1992): Betriebswirtschaftslehre. Band 1: Unternehmung und Umwelt, Marketing. - Zürich: Hans Schellenberg.

United Nations Development Programme, Civil Society Organizsations and Participation Programme (UNDP, CSOPP) (1998): Empowering People: A Guidebook to Participation. - http://www.undp.org/ csopp/CSO/NewFiles/docemppeople.html 28.8.2001.

UNESCO (Hrsg.) (1996): Biosphärenreservate. Die Sevilla-Strategie und die internationalen Leitlinien für das Weltnetz. - Bonn: Bundesamt für Naturschutz.

Walter, F. \& W. SPILlmanN (1999): Zwischenhalt auf dem Weg zum nachhaltigen Verkehr. - In: GAIA 8, 2: 93-101.

Walther, P. (2000): Biosphärenreservat Entlebuch: Schlussbericht der Aktionen zum Landschaftsschutz. Bern: Biosphärenreservat Entlebuch (Manuskript).

\section{Zusammenfassung: UNESCO Biosphäre Entlebuch: Modell für eine nachhaltige Regionalentwicklung? Konzept Zielerreichungskontrolle}

Das Entlebuch ist im September 2001 von der UNESCO als erstes Biosphärenreservat der Schweiz gemäss Sevilla-Strategie anerkannt worden. Biosphärenreservate sollen Modellregionen für eine nachhaltige Entwicklung sein. Um zu überprüfen, ob die UNESCO Biosphäre Entlebuch (UBE) dem Anspruch einer Modellregion tatsächlich gerecht wird, bedarf es regelmässiger Evaluationen. Dazu werden Schwerpunkte, Ziele und Indikatoren einer nachhaltigen Regionalentwicklung in partizipativen Prozessen her- geleitet, welche als Basis für eine künftige Zielerreichungskontrolle dienen werden.

Am Beispiel des Tourismus werden die wichtigsten inhaltlichen Resultate entlang des touristischen Leistungsbündels mit den Bausteinen Information, Anreise, Unterkunft, Verpflegung, Aktivitäten, Mobilität in der Region und Abreise sowie zu dessen Auswirkungen auf die drei Dimensionen einer nachhaltigen Entwicklung präsentiert.

Die konsequente Durchführung der Zielerreichungskontrolle wird zeigen, ob die UBE tatsächlich als Modell für eine nachhaltige Regionalentwicklung gelten kann.

\section{Summary: UNESCO Biosphere Reserve Entlebuch: Model for Sustainable Regional Development? Concept for Goal Achievement Assessment}

In September 2001, Entlebuch was recognised by the UNESCO as the first Biosphere Reserve in Switzerland defined according to the terms of the Seville Strategy. Biosphere reserves are expected to function as model regions for sustainable development. In order to verify whether the UNESCO Biosphere Reserve Entlebuch (UBE) fulfils the demands of a model region, periodical evaluations are necessary. For this purpose, a participatory process involving local interest groups was initiated in order to determine the main areas of development, goals and indicators. The results of these discussions will serve as the basis for the goal achievement assessment.

Focussing on tourism, this paper presents the most important results of the sustainable tourist service chain including the modules information, arrival, board and lodging, recreational activities, mobility in the region and departure, as well as the influence of this tourist service chain on the three dimensions of sustainable development.

The consequent implementation of goal achievement assessment will bring to light the real role model character of the UBE for regional sustainable development.

Résumé: «UNESCO Biosphère Entlebuch»: Modèle pour un développement régional durable? Concept «contrôle de réalisation des objectifs»

L'Entlebuch a été reconnu en septembre 2001 par l'UNESCO comme la première réserve de biosphère de la Suisse, conformément à la stratégie de Séville. Les réserves de biosphère doivent être des modèles régionaux pour un développement durable. Pour vérifier si cette réserve de biosphère correspond vraiment aux exigences d'une région modèle, il importe de mettre en œuvre de façon régulière des évaluations. A cet 
effet, il est fait recours à des buts et indicateurs d'un développement régional durable dans des processus participatifs, susceptibles de servir de base future à un contrôle des objectifs.

Les résultats essentiels sont fournis par l'exemple du tourisme, qui livre des données adéquates en matière d'information, d'arrivée, d'hébergement, de restauration, d'activités, de mobilité à l'intérieur d'une région, de départ et, en ce qui concerne les répercussions de ces services sur les trois dimensions d'un développement durable.

La réalisation pertinente du contrôle des objectifs va montrer si la réserve de l'UNESCO peut être considérée réellement comme un modèle de développement régional durable.

\section{Didaktische Hinweise}

- Welchen Anforderungen muss ein Biosphärenreservat entsprechen?

- Wie sind sie gegliedert und welchem Zweck dienen sie?

- Die Zonierung eines Biosphärenreservats (Kern-, Pflege- und Entwicklungszone) nach dem menschlichen Einfluss lässt sich auf kleinere Raumeinheiten übertragen: Wie würde eine Zonierung in der eigenen Region, Gemeinde oder gar rund ums Schulhaus aussehen?

- Welche Indikatoren ergeben Hinweise für eine erfolgreiche nachhaltige Regionalpolitik?
- Im vorliegenden Artikel wird das touristische Leistungsbündel aus Sicht der AnbieterInnen thematisiert. Lernende können ihrerseits als touristische NachfragerInnen betrachtet werden. Zum Einstieg können somit ihre Erwartungen bezüglich des touristischen Angebots in Grossschutzgebieten abgefragt werden. Diese lassen sich anschliessend mit den Resultaten der AnbieterInnen vergleichen und allfälliger Handlungsbedarf kann abgeleitet werden.

- Wie müsste eine nachhaltige Produktlinie für Käse, Holz oder andere Produkte aussehen?

- Welches sind die Kennzeichen nachhaltiger Regionalentwicklung?

Dr. Annette Schmid, Dr. Engelbert Ruoss, UNESCO Biosphäre Entlebuch, Chlosterbühl28, CH-6170 Schüpfheim.

e-mail: a.schmid@biosphaere.ch,e.ruoss@biosphaere.ch

Prof. Dr. Hans Elsasser, Geographisches Institut der Universität Zürich, Abteilung Wirtschaftsgeographie, Winterthurerstrasse 190, CH-8057 Zürich.

e-mail: elsasser@geo.unizh.ch

\section{Manuskripteingang/received/manuscrit entré le} 8.3.2004

Annahme zum Druck/accepted for publication/accepté pour l'impression: 24.5.2004 